\title{
Resistance to endocrine therapy in breast cancer: molecular mechanisms and future goals
}

\author{
Małgorzata Szostakowska ${ }^{1}$ Alicja Trębińska-Stryjewska ${ }^{1}$ Ewa Anna Grzybowska ${ }^{1}$ Anna Fabisiewicz ${ }^{1}$ (I)
}

Received: 20 August 2018 / Accepted: 20 October 2018 / Published online: 1 November 2018

(c) The Author(s) 2018

\begin{abstract}
Introduction The majority of breast cancers (BCs) are characterized by the expression of estrogen receptor alpha (ER $\alpha+)$. $\mathrm{ER} \alpha$ acts as ligand-dependent transcription factor for genes associated with cell survival, proliferation, and tumor growth. Thus, blocking the estrogen agonist effect on ER $\alpha$ is the main strategy in the treatment of ER $\alpha+\mathrm{BCs}$. However, despite the development of targeted anti-estrogen therapies for $\mathrm{ER}+\mathrm{BC}$, around $30-50 \%$ of early breast cancer patients will relapse. Acquired resistance to endocrine therapy is a great challenge in ER+ BC patient treatment.

Discussion Anti-estrogen resistance is a consequence of molecular changes, which allow for tumor growth irrespective of estrogen presence. Those changes may be associated with ER $\alpha$ modifications either at the genetic, regulatory or protein level. Additionally, the activation of alternate growth pathways and/or cell survival mechanisms can lead to estrogen-independence and endocrine resistance.

Conclusion This comprehensive review summarizes molecular mechanisms associated with resistance to anti-estrogen therapy, focusing on genetic alterations, stress responses, cell survival mechanisms, and cell reprogramming.
\end{abstract}

Keywords Breast cancer $\cdot$ Endocrine therapy $\cdot$ Resistance $\cdot$ Molecular mechanisms

\section{Introduction}

Breast cancer $(\mathrm{BC})$ is one of the most common female tumors diagnosed worldwide. In the U.S., BC represents $29 \%$ of all new cancer cases in women [1]. Metastatic breast malignancy is also the second most fatal cancer in woman.

In order to choose the most efficient treatment strategy, it is crucial to determine the biological subtype of the examined breast cancer. Biological subtypes are classified according to the expression of steroid receptors (estrogen (ER) and progesterone (PR)) and HER-2 (human epidermal

Anna Fabisiewicz

anna.fabisiewicz@coi.pl

Małgorzata Szostakowska

malgorzata.szostakowska@coi.pl

Alicja Trębińska-Stryjewska

alicja.trebinska@wat.edu.pl

Ewa Anna Grzybowska

ewa.grzybowska@coi.pl

1 Department of Molecular and Translational Oncology, The Maria Skłodowska-Curie Institute of Oncology, Roentgena 5, Warsaw, Poland growth factor receptor 2) established via immunohistochemistry (IHC) (Table 1). Cancers that are positive for estrogen receptor $(\mathrm{ER}+)$ and/or progesterone receptor $(\mathrm{PR}+)$ in IHC are classified as luminal. Currently, more accurate molecular expression profiling is available (PAM50 assay) that enables to distinguish more accurate risk groups (high recurrence risk subtypes) [2-4].

Luminal cancers have a better prognosis than other types of $\mathrm{BC}$ and are sensitive to anti-estrogen therapy $[10,11]$. They are further divided into subgroups (luminal A, B and C), which differ in aggressiveness. Luminal A breast cancers are characterized by high expression of luminal epithelial genes, low expression of $\mathrm{Ki}-67$ and distinct methylation profile of more than 40 gens (see review: [12]). The luminal B breast cancers are characterized by higher Ki-67 and lower expression of several luminal-related genes (like ESRI or FOXA1), genomic instability and a higher frequency of TP53 gene mutations; thus, they are associated with a worse prognosis and a higher risk of relapse than luminal A breast cancers [5, 12]. Luminal C subtype, characterized by molecular profiling and unrecognizable via IHC displays the overexpression of genes that are characteristic for 
Table 1 Biological subtypes of breast cancer distinguished by IHC

\begin{tabular}{lllllll}
\hline Biological subtype & ER & PR & HER-2 & Recommended I line treatment & Frequency & References \\
\hline Luminal A & + & $+/-$ & - & Endocrine therapy & $40-50 \%$ & {$[5-7]$} \\
& $(-)$ & $(+)$ & & & & $(<3 \%)$ \\
Luminal B & + & $+/-$ & $+/-$ & Endocrine therapy combined with chemotherapy & $20-30 \%$ \\
& $(-)$ & $(+)$ & & & & $(<3 \%)$ \\
HER-2 enriched & - & - & + & Anti-HER2, adjuvant therapy, chemotherapy & $20-30 \%$ & {$[5,8]$} \\
Basal-like (Triple-negative) & - & - & - & Systemic chemotherapy & $\sim 15 \%$ & {$[5,9]$} \\
\hline
\end{tabular}

non-luminal breast cancers, like transferrin receptor (CD71), $M Y B$, nuclear protein $\mathrm{P} 40, S Q L E$, and GGH [5].

Luminal breast cancers constitute the majority of diagnosed breast cancers (Table 1) [5]. Non-luminal breast cancers (HER2 positive, triple-negative breast cancer) have poorer prognosis than luminal cancers. HER2-positive cancers represent $20-30 \%$ (Table 1) of all diagnosed breast cancers and can be treated with anti-HER2 antibodies [5, 8]. Triple-negative breast cancer (TNBC; without ER, PR and HER 2 expression) represents nearly $15 \%$ (Table 1) of BCs and have short disease-specific survival and poor prognosis $[5,13]$.

The "gold standard" treatment of luminal breast cancer is anti-estrogen therapy. The aim of this treatment is to block the effect of estrogen at the receptor level (selective estrogen receptor modulators, SERM/selective estrogen receptor down-regulators, SERD) or by inhibiting estrogen production (aromatase inhibitors) [14-16].

Despite the high sensitivity of luminal tumors to endocrine therapy, $30-50 \%$ of early breast cancer patients will later relapse. Additionally, these cancers have a tendency to stay dormant, often for many years and metastasis can be triggered as late as 20 years after diagnosis [6, 17]. Resistance to therapy and distant metastases are the main causes of death in breast cancer patients [10].

\section{Estrogen receptors}

There are three major forms of physiological estrogens in females: estrone (E1), estradiol (E2, or 17 $\beta$-estradiol), and estriol (E3) [18]. The estrogen receptor acts as a liganddependent transcription factor and has two forms: $E R \alpha$ and ER $\beta$, encoded by the ESR 1 and ESR 2 genes, respectively [19]. ER $\alpha$ is a transcription factor for genes associated with cell survival, proliferation, and tumor growth (e.g., genes for insulin-like growth factor-1 receptor (IGF1R), cyclin D1, anti-apoptotic BCL-2 protein, vascular endothelial growth factor (VEGF) ) [20]. The phosphorylation of ER $\alpha$ has a profound impact on its activity and ER $\alpha$-regulated gene expression, as well as on cell growth, migration, and morphology [21]. While the role of ER $\alpha$ in tumorigenesis is crucial, the role of ER $\beta$ is still controversial [22]. It is believed that $\mathrm{ER} \beta$ has anti-proliferative properties; thus, it acts as an $\mathrm{ER} \alpha$ antagonist and is not expressed in breast cancer cell lines [23]. However, detailed validation of commonly used ER $\beta$ antibodies has demonstrated that some of these reagents either detect ER $\beta$ only in specific experimental conditions or lack any specificity for ER $\beta$ across multiple assays. Therefore, our current understanding of ER $\beta$ role in cancer may be not accurate [24] and should be revalidated. The newest study on ER $\beta+$ TNBC cell lines suggests that ER $\beta$ expression may be the prognostic factor for TNBC patients. In $E R \beta+$ cells, $E 2$ is an activator for $E R \beta$ signaling, which induces cystatins and results in inhibition of TGF- $\beta$ signaling pathway. This suppression results in decrease of TNBC cells invasiveness in vitro [25].

Both ER $\alpha$ and $E R \beta$ possess several functional domains: the N-terminal domain (NTD), the DNA binding domain (DBD), and the ligand binding domain (LBD) [26]. Moreover, there are two activation domains (AFs) that are part of the NTD and LBD functional domains (AF1 and AF2, respectively). Activation domains in the agonist conformation are involved in the recruitment of co-activators and corepressors for certain target genes.

Full length estrogen receptor alpha has $66-\mathrm{kDa}(\mathrm{ER} \alpha-$ $66)$, but has a few isoforms coded by alternatively spliced mRNAs, from which best known are ER $\alpha-46$ and ER $\alpha-36$ [27] (Fig. 1). ER $\alpha-46$ was found in over $70 \%$ of breast tumors and displayed variable expression levels, sometimes higher than ER $\alpha-66$. In this isoform alternative splicing results in the removal of $\mathrm{N}$-terminal AF1 transactivation domain [28]. If ER $\alpha-66$ and $E R \alpha-46$ are coexpressed, $E R \alpha-46$ acts as a competitive inhibitor of $E R \alpha$ AF1 [29]. ER $\alpha-36$ lacks both activation factor sequences (AF1, AF2), but the DNA-binding domain, part of the ligand-binding domain and the propensity for partial dimerization are retained in this variant; thus, it may recognize the ligand. It is hypothesized that ER $\alpha-36$ may primarily function as a membrane-based estrogen receptor to mediate membrane-initiated, non-genomic, estrogen signaling [27]. It was also shown that tamoxifen acts as an agonist on ER $\alpha-36$ in breast cancer cells, promoting stemness and contributing to hormone therapy resistance 


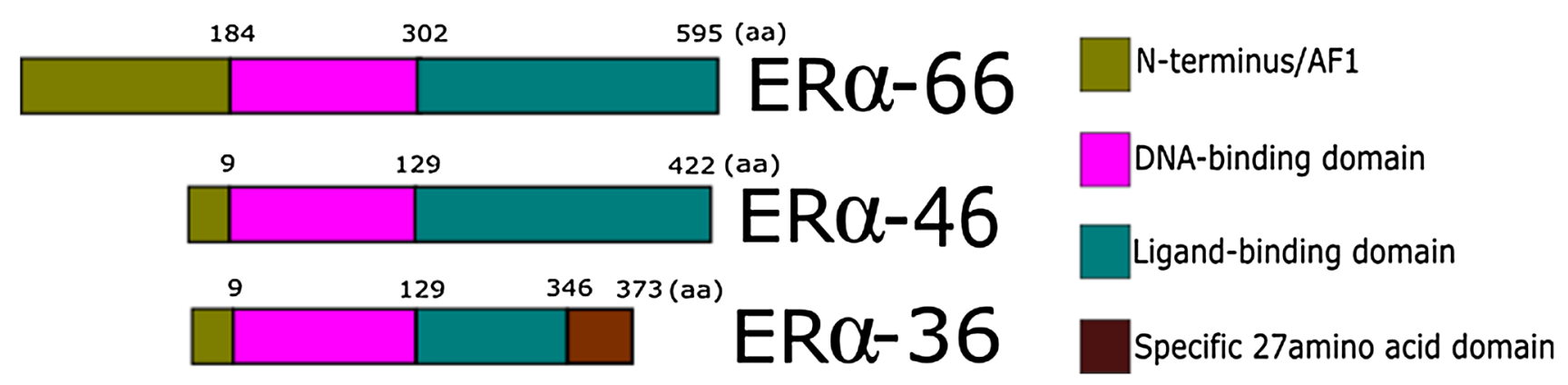

Fig. 1 Scheme of $E R \alpha, E R \alpha-46$, and $E R \alpha-36$ structure

and metastasis [30]. However, there are still no clinical data from trials taking into account ER $\alpha-36$ expression in $\mathrm{BC}$ patients.

\section{Resistance to endocrine therapy}

In $15-20 \%$ of cases, the resistance is associated with the activation of an ER $\alpha$-independent proliferation mechanism. It may be accompanied by a phenotypic change in cells, from $\mathrm{ER} \alpha+$ to $\mathrm{ER} \alpha-$. As luminal B breast cancers are characterized by the lower expression of ER $\alpha$ than luminal A breast cancers, there is a higher probability of $\mathrm{ER} \alpha$ expression loss in luminal $\mathrm{B}$ breast cancers [5, 31]. Therefore, luminal B breast cancers are considered to have a higher risk of acquiring endocrine resistance than luminal A breast cancers.

This review will focus on molecular mechanisms of estrogen-independence, ER switch and the role of miRNA, autophagy and stemness in acquiring resistance to endocrine therapy.

\section{ESR1 modifications}

As ER $\alpha$ (in this review referred to as 'ER') is the main target in anti-estrogen therapy, changes in ESRI gene may lead to estrogen-independence [32].

\section{Mutations}

One of the activation domains of estrogen receptor (AF2) is located at the end of the ligand-binding domain (LBD) and consists of four helices (H9-H12). Those helices are flexible, and their conformation is dependent on the ligand [33]. The crucial structural element for estrogen-dependent AF2 activity is an $\alpha$-helix, named 'helix 12' (H12). When LBD is bound to the E2, helix 12 unveils the binding place for ER co-activators. However, when bound with SERM or SERD, helix 12 changes its orientation and covers the binding place for ER co-activators [34, 35] (Fig. 2). The length of helix
A

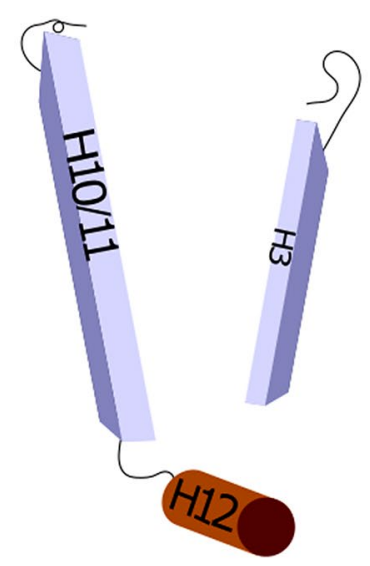

Fig. 2 Structure and conformation of helix 12 (H12) of the ER LBD. Scheme shows a fragment of the full ER protein and rollers stand for $\alpha$-helix structures. a Position of $\mathrm{H} 12$ when E2 or SERD/SERM is not bound to ER; b Position of H12 in antagonist ER conformation;
B

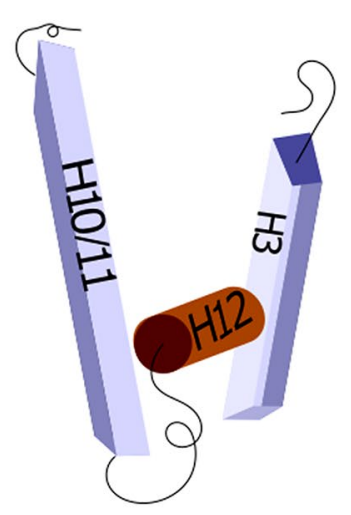

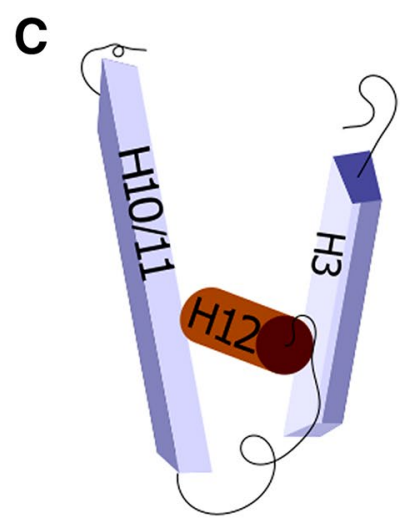

c Position of H12 in agonist ER conformation. Missense mutations cause conformational changes placing $\mathrm{H} 12$ in position of agonist conformation. Place and amino acid of substitution determines bond straight and stability of $\mathrm{H} 12$ position 
12 is different for agonist- and antagonist-bound structures; agonist-bound structures start at D538 and antagonist-bound structures at L536 [34]. Substitutions of amino acids: L536, Y537, D538 result in conformational changes, placing H12 in the agonist position. This leads to constitutive transactivation activity in the absence of a ligand. The stability and activity of these mutants are dependent on H11-H12 interaction. Strength of this interaction depends on the amino acid that replaced wild-type occurring amino acid [34-37]. E380Q, another substitution associated with $\mathrm{H} 12$ positioning, is placed away from H12 sequence, but is close to the carboxy-terminal portion of H12. In wild-type, glutamic acid (E) induces the repulsion between $\mathrm{H} 5$ and $\mathrm{H} 12$ that unfavorites the generation of agonist H12 position. Substitution to glutamine (Q) abolishes that repulsion, which allows H12 to adopt the agonist position without the E2 [38].

The point missense mutations in ESRl gene (Table 2) are associated with the constant ER activity. However, the clinical value of those single nucleotide variations (SNVs) remains debatable. The ESRI mutations are infrequent in the primary tumor, but their frequency in metastatic lesion may be even 30-times higher [39]. Meta-analysis of the clinical value of ESR1 mutations detected in cfDNA (cell-free DNA) revealed that they may have prognostic significance and clinical value in guiding further endocrine therapy choice in $\mathrm{ER}+\mathrm{MBC}$ patients who received prior AIs therapy, but not for patients treated with fulvestrant [40].

\section{Translocation}

Recent studies on breast cancer cell lines and patient-derived xenografts revealed a resistance-associated translocation of ESR1, ESR1/YAP1 (t $(6 ; 11))$ [44, 45]. The break point of $E S R l$ is located at the beginning of helix 12. The fusion protein consists of the N-terminal and DNA binding domains of ER and the C-terminus from YAP1 [44]. YAP1 is the nuclear effector in the HIPPO pathway, which is involved

Table 2 Most common missense mutations in ESR1 gene

\begin{tabular}{llll}
\hline $\begin{array}{l}\text { ESR1 point muta- } \\
\text { tion }\end{array}$ & Substitution & $\begin{array}{l}\text { Frequency in } \\
\text { BC patients } \\
(\%)\end{array}$ & References \\
\hline $1609 \mathrm{~T}>\mathrm{A}$ & $\begin{array}{c}\text { Y537N } \\
\text { (Tyr537Asn) }\end{array}$ & $5-33$ & {$[37,41]$} \\
$1610 \mathrm{~A}>\mathrm{C}$ & $\begin{array}{l}\text { Y537S } \\
\text { (Tyr537Ser) }\end{array}$ & $13-22$ & {$[36,41]$} \\
$1613 \mathrm{~A}>\mathrm{G}$ & $\begin{array}{c}\text { D538G } \\
\text { (Asp538Gly) }\end{array}$ & $14-36$ & {$[41-43]$} \\
$1608 \mathrm{TC}>\mathrm{AG}$ & $\begin{array}{c}\text { L536Q } \\
(\text { Leu536Gln })\end{array}$ & $<5$ & {$[41]$} \\
$1138 \mathrm{G}>\mathrm{C}$ & $\begin{array}{c}\text { E380Q } \\
\text { (Glu380Gln) }\end{array}$ & $<7$ & {$[43]$} \\
& & & \\
\hline
\end{tabular}

in cell development and growth. YAPI is one of the crucial genes in the regulation of proliferation, apoptosis, and stemness. Moreover, overexpression of this gene in breast cancer is associated with the promotion of proliferation, invasiveness, and cell survival [46]. Functional properties of the fusion protein include the following: estrogen-independent growth, constitutive expression of ER target genes, antiestrogen resistance, activation of a metastasis-associated transcriptional program, and inducing the cellular motility [47]. Newest studies identified multiple 3 ' partners for ESRI rearrangement, including $S O X 9, D A B 2, G Y G 1, M T H F D 1 L$, and PLEKHG1, CCDC170 [48, 49]. The cluster junctions for ESR 1 remain similar to the ESR I/YAP translocation, placed between exons 6 and 7. Activity of fusion protein is independent on the estrogen presence, but its level seems to be dependent on $3^{\prime}$ partner. For example, fusion with $S O X 9$ or $D A B 2$ results in 40 times higher activity of fusion protein than wild-type ER, while fusion with GYG1 results in 2-3 times higher activity than wild-type ER. While it is undoubtable that ESRI fusions are demonstrating constitutive, ligand-independent activity [49], there is still lack of evidence for clinical utility of described ESRI alterations.

\section{Amplification}

The amplification of ESR1 was widely studied [50-56]; nonetheless there are still controversial data about its frequency and prognostic value. Amplification frequency detected in breast cancer patients varies from 1 to $37 \%$ [50-54, 56]. The association of ESRI amplification and antiestrogen resistance is also discussed. Some studies report that ESR1 amplification correlates with tamoxifen resistance and shorter disease-free survival $[55,57]$, while other studies showed no correlation $[54,58]$.

\section{miRNAs as regulators of resistance to endocrine therapy}

The up-regulation of some miRNAs can be associated with anti-estrogen resistance, either mediated by the activation of alternate growth pathways or by the inhibition of ER expression. miR-155, miR-221/222, miR-21, miR-125b are assumed to be key upregulated miRNAs in a breast cancer resistance to different types of treatment (see review: [59]). Yet miR-155 and miR-221/222 seem to be most associated with resistance to antiestrogen therapy. miR-155 targets SOC6 (inhibitor cytokine signaling) and stimulates the activation of STAT3 signaling pathway. This pathway is associated with cell survival and resistance to tamoxifen $[59,60]$. miR-221 and miR-222 were found to regulate various oncogenic pathways and their high expression was found to be highly correlated with tamoxifen resistance [61, 62]. MCF-7 cells transfected with miR-221/222 overexpress components 
of TGF- $\beta$ and TP53 signaling pathways. Also, these miRNAs up-regulate the transcriptional activity of $\beta$-catenin and the expression of the Wnt pathway proteins: WNT5A and FZD5. At the same time, miR-221/222 inhibit the expression of P27 and Wnt pathway inhibitors: AXIN2, SFRP2, CHD8, and NLK [61-63]. The role of Wnt pathway deregulation in cancer development is well known (reviewed: [64, 65]). P27 is cycle-dependent kinase inhibitor (CDKI) from the CIP/KIP family of CDKIs. The inhibition of P27 drives cell proliferation by relieving G1 arrest [66]. This deregulation of the cell cycle is found in ER+ breast cancer cell lines resistant to tamoxifen $[61,62]$. The expression level of P27 was found to be predictive for patients treated with tamoxifen [67]. Furthermore, exosomes with miR-221/222 can be transferred from resistant cells to sensitive cells, promoting tamoxifen resistance [61].

The down-regulation of some microRNAs can also be associated with endocrine resistance. The low expression of the ER $\alpha-36$ inhibitors miR-210 and let-7 [68, 69] leads to overexpression of this isoform and ER $\alpha-36$-mediated anti-estrogen resistance. Let-7 miRNAs match the 3421 to 3442 region located in the $3^{\prime} \mathrm{UTR}$ of the ER $\alpha-36$ coding transcript, inducing mRNA degradation and ER $\alpha-36$ downregulation [69]. ER $\alpha-36$ was found to be an $\mathrm{ER} \beta$ antagonist and agonist of estrogen-like SERM effects [70]. The high expression of this ER isoform is observed in TNBC and luminal cancers resistant to endocrine therapy with an ER expression switch [71]. This may suggest that the loss of ER expression in luminal BCs could be associated with the increased production of ER $\alpha-36$. In vitro studies confirmed that the low expression of let-7 miRNAs results in ER $\alpha$ 36-mediated endocrine resistance, whereas the restoration of let-7 miRNAs expression leads to a loss of tamoxifen resistance in the MCF-7 tamoxifen-resistant cell line [69]. Another miRNA, miR-873 is involved in the phosphorylation and activation of ER. A direct target of miR-873 is cycle-dependent kinase 3 (CDK3), which phosphorylates ER at Ser 104, 106, and 118, leading to enhanced transcriptional activity of ER. Overexpression of CDK3 in breast cancer is associated with increased cell proliferation and resistance to endocrine therapy. The restoration of miR-873 expression results in the decreased activity of ER and sensitizes breast cancer cells to tamoxifen in vivo [68]. Due to the effect of restoring abovementioned miRNAs expression on tamoxifen resistance, they are considered to be used in future target therapies for patients with resistant breast cancers.

\section{Unfolded protein response (UPR)}

Unfolded protein response (UPR) is a sensor system for endoplasmic reticulum (EnR) stress caused by the accumulation of unfolded or misfolded proteins. UPR signaling was show to regulate both autophagy and apoptosis [72]. There are three main arms of UPR: (1) autophosphorylation of protein kinase RNA-like endoplasmic reticulum kinase (PERK), which leads to transient inhibition of protein synthesis; (2) proteolytic cleavage and activation of transcription factor ATF6 $\alpha$ and (3) oligomerization and autophosphorylation of inositol-requiring enzyme 1 (IRE1 $\alpha$ ), which controlsalternative splicing of XBP1 mRNA (X-box binding protein 1). Spliced variant of XBP1 may act as transcriptional activator for BCL2 [73], which is known as an anti-apoptotic protein and one of the autophagy suppressors [74]. UPR is sensitive to intracellular calcium concentration: in the absence of stress, the three UPR molecular sensors are maintained inactive through the association with calcium-dependent chaperone; depleted calcium stores in endoplasmic reticulum cause the removal of this chaperone from sensors, leading to UPR activation. Subsequent steps include induction of protein-folding chaperones, resulting in increased folding and protein degradation.

Two different modes of UPR were described: "reactive"-classic response to EnR stress and "anticipatory"a hormone-dependent pathway in which cells mildly preactivate the UPR [75]. Mild activation of UPR results in enhanced resistance to stress, representing survival advantage, while strong, sustained activation leads to cell death. It has been shown that hormonal activation of UPR occurs without accumulation of unfolded proteins and involves opening of EnR calcium channels. Among UPR-activating steroid hormones, E2 is prominent. There is a strong correlation between the expression of UPR gene signature and the expression of estrogen regulated genes [76]. Moreover, a strong correlation of UPR gene signature with subsequent resistance to tamoxifen therapy was observed in ER+ breast cancer patients [76]. It was demonstrated that either E2 or competitor antiestrogens and aromatase inhibitors activate mild, pro-survival UPR [77].

\section{Autophagy}

Autophagy is an essential process for homeostasis and the survival of eukaryotic cells. This process eliminates damaged or old organelles, and removes long-lived proteins and/ or protein aggregates; thus, it is responsible for the quality control of essential cellular components [78]. Mechanisms of autophagy regulation are well known (reviewed: [79]) and involve unfolded protein response (UPR), phosphatidylinositol 3-kinase (PtdIns3K, PI3K), mammalian target of rapamycin (mTOR) and glucose-dependent protein 78 (GPR78) [80].

In breast cancer cells, autophagy was identified as an initiator of activated cell death induced by anti-estrogen drugs (ACD II) [81]. However, recent studies have recognized autophagy as a survival mechanism. While ACD II is associated with endoplasmic reticulum stress, autophagy 
allows cells to survive under this condition [78]. Although the increased activation of autophagy is found in cells after anti-estrogen treatment, only $20-30 \%$ of MCF-7 population undergo apoptosis [82]. The detailed mechanism of autophagy-mediated resistance is still not clear, there is a strong evidence that the inhibition of autophagy increases sensitivity of cancer cells to anti-estrogen treatment [82-84]. In vivo models showed that autophagy inhibitors restore antiestrogen sensitivity in resistant tumors [85].

\section{Mammalian target of rapamycin (mTOR)}

Mammalian target of rapamycin (mTOR) kinase is the component of two mTOR complexes: mTORC1 and mTORC2. mTORC1 is a major downstream target of the PI3K/AKT pathway and a negative regulator of autophagy. mTORC1 complex phosphorylates ULK1/2 and ATG13 in nutrientrich conditions, leading to the deactivation of these complexes. In starvation and stress conditions, the expression of mTORC 1 is decreased, and ULK1/2 and ATG 13 complexes are partially dephosphorylated. This process activates these proteins and enables the formation of a ULK1-ATG13FIP200 complex, which is essential for autophagosome formation [86] (Fig. 3). The down-regulation of mTORC1 by rapamycin or PI3K/AKT signaling inhibition leads to an increase in autophagy and can support the acquisition

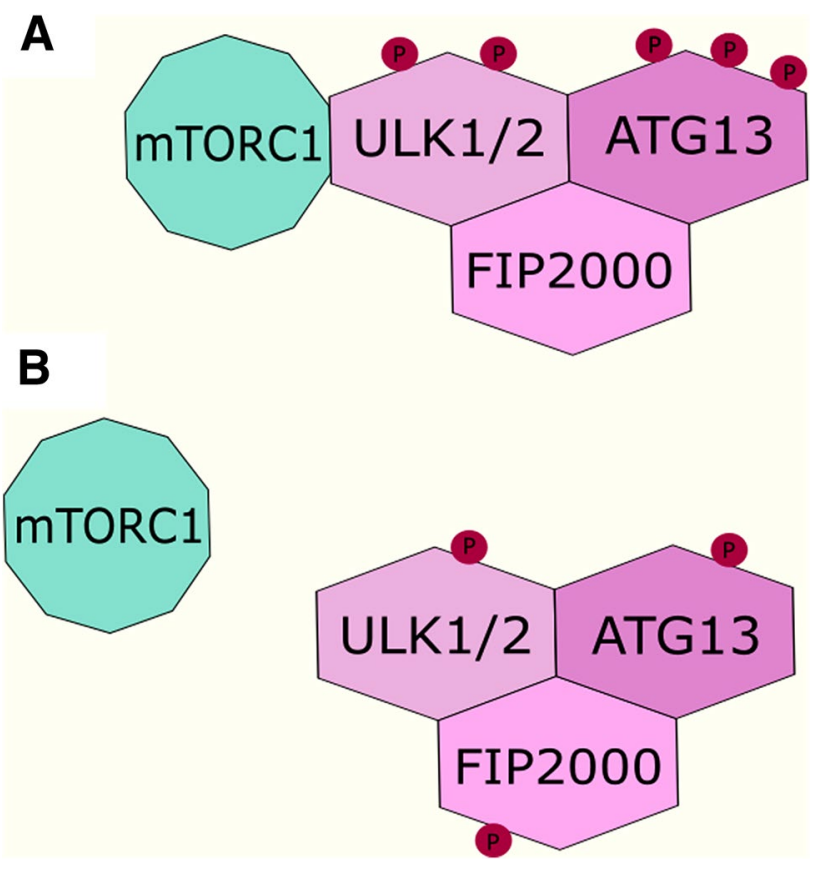

Fig. 3 Scheme of regulation of ULK1/2-ATG13-FIP200 complex by mTORC1. a mTORC1 phosphorylates ULK1/2 and ATG13 in nutrient-rich conditions; formation of the autophagosomes is suppressed. b mTORC1 detaches from complex; ULK1/2 and ATG13 are partially dephosphorylated; induced formation of autophagosomes of resistance to tamoxifen in breast cancer cells. Despite the insulin/growth factor and PI3K/AKT pathway being a negative regulators of autophagy, their hyperactivation is well characterized as an anti-estrogen resistance mechanism (reviewed: [87, 88]). Clinical trials with the use of PI3K/ AKT inhibitors combined with aromatase inhibitors revealed that PI3K/AKT inhibitors were efficient only in patients with identified PI3K/AKT hyperactivation [89]. In fact, those inhibitors combined with endocrine therapy may be detrimental for some patients. Activation of the PI3K/AKT pathway in stroma is crucial for stroma-associated tumor regression. An in vivo study highlighted that in cancers with low basal expression of PI3K pathway components, treatment with PI3K/AKT inhibitors impaired this process [90].

\section{Cancer stem-like cells}

Cancer stem-like cells (CSCs) are a small population of tumor cells that are capable of self-renewal, producing heterogeneous lineages of cancer cells that comprise the tumor. CSCs are a unique biological subpopulation of cancer cells that could survive indeterminately [91]. Those cells are characterized by the expression of gene sets associated with embryonic stem cells, the activation of multidrug resistance (MDR) and/or DNA-damage response (DDR) systems [92, 93].

Although detailed processes of the formation of cancer stem-like cells are still under debate, it is certain that cancer cells must go through the process of cell reprogramming. This process resets differentiated cells to a pluripotent state and can be achieved by nuclear transfer, cell fusion and/or overexpression of certain transcription factors: $O c t-4$, Sox2, Klf4, c-Myc (OSKM) [94] (human homologs: POU5F1, SOX2, KLF4, MYC). As CSCs overexpress SOX2, POU5F1, and $N A N O G$, it is suspected that reprogramming of cancer cells is mediated via overexpression of those transcription factors [95]. From stemness markers, SOX2 is best known for its association with anti-estrogen resistance. In vitro studies showed that in ER- endocrine resistant MCF-7 cells SOX2 expression is significantly higher than in ER+resistant MCF-7 cells [95]. Other in vitro studies revealed that in ER+ endocrine resistant cell lines, SOX2 expression level is negatively correlated with ER and PR expression level. Interestingly, the high expression of SOX2 was also correlated with increasing histopathological grade during tamoxifen resistance acquisition [96]. There is growing evidence that SOX2 expression is highly correlated with resistance mechanisms and epithelial-mesenchymal transition (EMT)-specific gene expression in cancers. $S O X 2$ is the regulator of: GLI1, FOXA1, mTOR, EGFR, and WNT and/ or NF- $\mathrm{kB}$ pathway genes expression [95-100]. Those pathways are associated with growth, proliferation, dedifferentiation and resistance in cancer cells. Moreover, overexpression 
of other stemness markers can lead to hyperactivation of migration, survival and proliferation associated pathways: HEDGEHOG, WNT, NF- $\kappa$ B, TGF- $\beta$, NOTCH, ERK/MAPK (reviewed: [64, 101-103]). Activation of these pathways can lead to the acquisition of anti-estrogen resistance, increased invasiveness, migration and formation of distant metastases.

\section{Summary and future goals}

As described in this review, there are multiple anti-estrogen resistance mechanisms in breast cancer cells, which pose a great challenge in $\mathrm{BC}$ treatment. It seems plausible that some of those resistance pathways and systems are linked, which must be taken into account when designing new treatments. For example, PI3K inhibitors impair the stroma-mediated tumor regression of $\mathrm{BC}$ in mouse models [90]. Moreover, these inhibitors can promote the activation of cell survival mechanisms related to endocrine resistance, like autophagy. There is a need for a better understanding of the plausible cross-regulation of different resistance-associated molecular mechanisms, to improve the development of targeted drugs. Furthermore, it is essential to validate the detection techniques, prognostic and value of molecular mechanisms described above.

Funding National Science Center of Poland, Award Number: 2016/21/B/NZ2/03473

\section{Compliance with ethical standards}

Conflict of interest The authors declare to have no conflict of interest.

Ethical approval This article does not contain any interventional studies with human participants or animals performed by any of the authors.

Open Access This article is distributed under the terms of the Creative Commons Attribution 4.0 International License (http://creativeco mmons.org/licenses/by/4.0/), which permits unrestricted use, distribution, and reproduction in any medium, provided you give appropriate credit to the original author(s) and the source, provide a link to the Creative Commons license, and indicate if changes were made.

\section{References}

1. Siegel RL, Jemal MK, Jamel A (2016) Cancer statistics, 2016. CA 66(1):7-30

2. Nielsen T et al (2014) Analytical validation of the PAM50based prosigna breast cancer prognostic gene signature assay and nCounter analysis system using formalin-fixed paraffinembedded breast tumor specimens. BMC Cancer 14:177

3. Wallden B et al (2015) Development and verification of the PAM50-based Prosigna breast cancer gene signature assay. BMC Med Genom 8:54
4. Parker JS et al (2009) Supervised risk predictor of breast cancer based on intrinsic subtypes. J Clin Oncol 27(8):1160-1167

5. Kittaneh M, Montero AJ, Gluck S (2013) Molecular profiling for breast cancer: a comprehensive review. Biomark Cancer 5:61-70

6. Ignatiadis M, Sotiriou C (2013) Luminal breast cancer: from biology to treatment. Nat Rev Clin Oncol 10(9):494-506

7. Itoh $\mathrm{M}$ et al (2014) Estrogen receptor (ER) mRNA expression and molecular subtype distribution in ER-negative/progesterone receptor-positive breast cancers. Breast Cancer Res Treat 143(2):403-409

8. Jelovac D, Wolff AC (2012) The adjuvant treatment of HER2positive breast cancer. Curr Treat Options Oncol 13(2):230-239

9. Sharma P (2016) Biology and management of patients with triple-negative breast cancer. Oncologist 21(9):1050-1062

10. Matsumoto A et al (2015) Prognostic implications of receptor discordance between primary and recurrent breast cancer. Int $\mathbf{J}$ Clin Oncol 20(4):701-708

11. Ma CX, Sanchez CG, Ellis MJ (2009) Predicting endocrine therapy responsiveness in breast cancer. Oncology (Williston Park) 23(2):133-142

12. Dumitrescu RG (2018) Interplay between genetic and epigenetic changes in breast cancer subtypes. Methods Mol Biol 1856:19-34

13. Nielsen $\mathrm{TO}$ et al (2004) Immunohistochemical and clinical characterization of the basal-like subtype of invasive breast carcinoma. Clin Cancer Res 10(16):5367-5374

14. Kuiper GG et al (1997) Comparison of the ligand binding specificity and transcript tissue distribution of estrogen receptors alpha and beta. Endocrinology 138(3):863-870

15. Robertson JF et al (2014) A good drug made better: the fulvestrant dose-response story. Clin Breast Cancer 14(6):381-389

16. Lambertini $\mathrm{M}$ et al (2015) Ovarian suppression using luteinizing hormone-releasing hormone agonists during chemotherapy to preserve ovarian function and fertility of breast cancer patients: a meta-analysis of randomized studies. Ann Oncol 26(12):2408-2419

17. Zhang XH et al (2013) Metastasis dormancy in estrogen receptorpositive breast cancer. Clin Cancer Res 19(23):6389-6397

18. Cui J, Shen Y, Li R (2013) Estrogen synthesis and signaling pathways during aging: from periphery to brain. Trends Mol Med 19(3):197-209

19. Paterni I et al (2014) Estrogen receptors alpha (ERalpha) and beta (ERbeta): subtype-selective ligands and clinical potential. Steroids 90:13-29

20. Arpino $\mathrm{G}$ et al (2008) Crosstalk between the estrogen receptor and the HER tyrosine kinase receptor family: molecular mechanism and clinical implications for endocrine therapy resistance. Endocr Rev 29(2):217-233

21. Anbalagan M, Rowan BG (2015) Estrogen receptor alpha phosphorylation and its functional impact in human breast cancer. Mol Cell Endocrinol 418 Pt 3:264-272

22. Speirs V, Carder PJ, Lansdown MR (2002) Oestrogen receptor beta: how should we measure this? Br J Cancer 87(6):687; author reply $688-9$

23. Haldosen LA, Zhao C, Dahlman-Wright K (2014) Estrogen receptor beta in breast cancer. Mol Cell Endocrinol 382(1):665-672

24. Nelson AW et al (2017) Comprehensive assessment of estrogen receptor beta antibodies in cancer cell line models and tissue reveals critical limitations in reagent specificity. Mol Cell Endocrinol 440:138-150

25. Reese JM et al. (2018) ERbeta-mediated induction of cystatins results in suppression of TGFbeta signaling and inhibition of triple-negative breast cancer metastasis. Proc Natl Acad Sci USA 115(41):E9580-E9589 
26. Kumar R, Thompson EB (1999) The structure of the nuclear hormone receptors. Steroids 64(5):310-319

27. Wang $\mathrm{Z}$ et al (2005) Identification, cloning, and expression of human estrogen receptor-alpha36, a novel variant of human estrogen receptor-alpha66. Biochem Biophys Res Commun 336(4):1023-1027

28. Chantalat E et al (2016) The AF-1-deficient estrogen receptor ERalpha46 isoform is frequently expressed in human breast tumors. Breast Cancer Res 18(1):123

29. Flouriot $\mathrm{G}$ et al (2000) Identification of a new isoform of the human estrogen receptor-alpha (hER-alpha) that is encoded by distinct transcripts and that is able to repress hER-alpha activation function 1. EMBO J 19(17):4688-4700

30. Wang Q et al (2018) Tamoxifen enhances stemness and promotes metastasis of ERalpha36(+) breast cancer by upregulating ALDH1A1 in cancer cells. Cell Res 28(3):336-358

31. Osborne CK, Schiff R (2011) Mechanisms of endocrine resistance in breast cancer. Annu Rev Med 62:233-247

32. Jeselsohn R et al (2015) ESR1 mutations-a mechanism for acquired endocrine resistance in breast cancer. Nat Rev Clin Oncol 12(10):573-583

33. Pike AC (2006) Lessons learnt from structural studies of the oestrogen receptor. Best Pract Res Clin Endocrinol Metab 20(1):1-14

34. Skafar DF (2000) Formation of a powerful capping motif corresponding to start of "helix 12" in agonist-bound estrogen receptor-alpha contributes to increased constitutive activity of the protein. Cell Biochem Biophys 33(1):53-62

35. Merenbakh-Lamin K et al (2013) D538G mutation in estrogen receptor-alpha: A novel mechanism for acquired endocrine resistance in breast cancer. Cancer Res 73(23):6856-6864

36. Weis KE et al (1996) Constitutively active human estrogen receptors containing amino acid substitutions for tyrosine 537 in the receptor protein. Mol Endocrinol 10(11):1388-1398

37. Zhang QX et al (1997) An estrogen receptor mutant with strong hormone-independent activity from a metastatic breast cancer. Cancer Res 57(7):1244-1249

38. Katzenellenbogen JA et al (2018) Structural underpinnings of oestrogen receptor mutations in endocrine therapy resistance. Nat Rev Cancer 18(6):377-388

39. De Mattos-Arruda L et al (2014) Capturing intra-tumor genetic heterogeneity by de novo mutation profiling of circulating cell-free tumor DNA: a proof-of-principle. Ann Oncol 25(9):1729-1735

40. Zhang K et al (2018) Clinical value of circulating ESR1 mutations for patients with metastatic breast cancer: a meta-analysis. Cancer Manag Res 10:2573-2580

41. Pavlin M et al (2018) A computational assay of estrogen receptor alpha antagonists reveals the key common structural traits of drugs effectively fighting refractory breast cancers. Sci Rep 8(1):649

42. Toy W et al (2013) ESR1 ligand-binding domain mutations in hormone-resistant breast cancer. Nat Genet 45(12):1439-1445

43. Fribbens $C$ et al (2016) Plasma ESR1 mutations and the treatment of estrogen receptor-positive advanced breast cancer. J Clin Oncol 34(25):2961-2968

44. Li S et al (2013) Endocrine-therapy-resistant ESR1 variants revealed by genomic characterization of breast-cancer-derived xenografts. Cell Rep 4(6):1116-1130

45. Piccart M, Chen RH, Campone D, Burris M, Taran HA, Sahmoud T, Deleu T, Hortobagyi I, Baselga G (2013) J., Assessment of genetic alterations in postmenopausal women with hormone receptor-positive, HER2-negative advanced breast cancer from the bolero-2 trial by next-generation sequencing. Ann Oncol 24(supplement 3):25-26
46. Shi P, Chen FJ, Chen C (2015) Hippo pathway in mammary gland development and breast cancer. Acta Biochim Biophys Sin (Shanghai) 47(1):53-59

47. Lei JT et al (2018) Functional annotation of ESR1 gene fusions in estrogen receptor-positive breast cancer. Cell Rep 24(6):14341444 e7

48. Veeraraghavan J et al (2014) Recurrent ESR1-CCDC170 rearrangements in an aggressive subset of oestrogen receptor-positive breast cancers. Nat Commun 5:4577

49. Hartmaier RJ et al (2018) Recurrent hyperactive ESR1 fusion proteins in endocrine therapy-resistant breast cancer. Ann Oncol 29(4):872-880

50. Holst F et al (2007) Estrogen receptor alpha (ESR1) gene amplification is frequent in breast cancer. Nat Genet 39(5):655-660

51. Brown LA et al (2008) ESR1 gene amplification in breast cancer: a common phenomenon? Nat Genet 40(7):806-807, author reply $810-2$

52. Reis-Filho JS et al. (2008) ESR1 gene amplification in breast cancer: a common phenomenon? Nat Genet 40(7):809-810, author reply $810-2$

53. Horlings $\mathrm{HM}$ et al (2008) ESR1 gene amplification in breast cancer: a common phenomenon? Nat Genet 40(7):807-808, author reply $810-2$

54. Tomita $\mathrm{S}$ et al (2009) Estrogen receptor alpha gene ESR1 amplification may predict endocrine therapy responsiveness in breast cancer patients. Cancer Sci 100(6):1012-1017

55. Nielsen KV et al (2011) Amplification of ESR1 may predict resistance to adjuvant tamoxifen in postmenopausal patients with hormone receptor positive breast cancer. Breast Cancer Res Treat 127(2):345-355

56. Moelans CB et al (2013) ESR1 amplification in breast cancer by optimized RNase FISH: frequent but low-level and heterogeneous. PLoS One 8(12):e84189

57. Lin CH et al (2013) Clinical significance of ESR1 gene copy number changes in breast cancer as measured by fluorescence in situ hybridisation. J Clin Pathol 66(2):140-145

58. Vincent-Salomon A et al (2008) ESR1 gene amplification in breast cancer: a common phenomenon? Nat Genet 40(7):809

59. Campos-Parra AD et al (2017) Micro-RNAs as potential predictors of response to breast cancer systemic therapy: future clinical implications. Int J Mol Sci 18(6): 1182

60. Shen $\mathrm{R}$ et al (2015) MiRNA-155 mediates TAM resistance by modulating SOCS6-STAT3 signalling pathway in breast cancer. Am J Transl Res 7(10):2115-2126

61. Wei Y et al (2014) Exosomal miR-221/222 enhances tamoxifen resistance in recipient ER-positive breast cancer cells. Breast Cancer Res Treat 147(2):423-431

62. Miller TE et al (2008) MicroRNA-221/222 confers tamoxifen resistance in breast cancer by targeting p27Kip1. J Biol Chem 283(44):29897-29903

63. Rao X et al (2011) MicroRNA-221/222 confers breast cancer fulvestrant resistance by regulating multiple signaling pathways. Oncogene 30(9):1082-1097

64. Yu QC, Verheyen EM, Zeng YA (2016) Mammary development and breast cancer: a Wnt perspective. Cancers (Basel) 8(7):65

65. Polakis P (2012) Wnt signaling in cancer. Cold Spring Harbor Perspect Biol 4(5):a008052

66. Quereda V, Malumbres M (2009) Cell cycle control of pituitary development and disease. J Mol Endocrinol 42(2):75-86

67. Stendahl M et al (2010) p27Kip1 is a predictive factor for tamoxifen treatment response but not a prognostic marker in premenopausal breast cancer patients. Int J Cancer 127(12):2851-2858

68. Cui J et al (2015) MiR-873 regulates ERalpha transcriptional activity and tamoxifen resistance via targeting CDK3 in breast cancer cells. Oncogene 34(30):3895-3907 
69. Zhao Y et al (2011) let-7 microRNAs induce tamoxifen sensitivity by downregulation of estrogen receptor alpha signaling in breast cancer. Mol Med 17(11-12):1233-1241

70. Wang $\mathrm{Z}$ et al (2006) A variant of estrogen receptor- $\{$ alpha $\}$, hER\{alpha\}36: transduction of estrogen- and antiestrogen-dependent membrane-initiated mitogenic signaling. Proc Natl Acad Sci USA 103(24):9063-9068

71. Pelekanou V et al (2012) ERalpha36, a new variant of the ERalpha is expressed in triple negative breast carcinomas and has a specific transcriptomic signature in breast cancer cell lines. Steroids 77(10):928-934

72. Clarke R, Cook KL (2015) Unfolding the role of stress response signaling in endocrine resistant breast cancers. Front Oncol 5:140

73. Clarke R et al (2012) Endoplasmic reticulum stress, the unfolded protein response, autophagy, and the integrated regulation of breast cancer cell fate. Cancer Res 72(6):1321-1331

74. Cook KL, Shajahan AN, Clarke R (2011) Autophagy and endocrine resistance in breast cancer. Expert Rev Anticancer Ther 11(8):1283-1294

75. Shapiro DJ et al (2016) Anticipatory UPR activation: a protective pathway and target in cancer. Trends Endocrinol Metab 27(10):731-741

76. Andruska $\mathrm{N}$ et al (2015) Anticipatory estrogen activation of the unfolded protein response is linked to cell proliferation and poor survival in estrogen receptor alpha-positive breast cancer. Oncogene 34(29):3760-3769

77. Cook KL et al (2014) Knockdown of estrogen receptor-alpha induces autophagy and inhibits antiestrogen-mediated unfolded protein response activation, promoting ROS-induced breast cancer cell death. FASEB J 28(9):3891-3905

78. Yang Z, Klionsky DJ (2010) Mammalian autophagy: core molecular machinery and signaling regulation. Curr Opin Cell Biol 22(2):124-131

79. He C, Klionsky DJ (2009) Regulation mechanisms and signaling pathways of autophagy. Annu Rev Genet 43:67-93

80. Cook KL et al (2012) Glucose-regulated protein 78 controls cross-talk between apoptosis and autophagy to determine antiestrogen responsiveness. Cancer Res 72(13):3337-3349

81. Bursch W et al (1996) Active cell death induced by the anti-estrogens tamoxifen and ICI 164384 in human mammary carcinoma cells (MCF-7) in culture: the role of autophagy. Carcinogenesis 17(8):1595-1607

82. Samaddar JS et al (2008) A role for macroautophagy in protection against 4-hydroxytamoxifen-induced cell death and the development of antiestrogen resistance. Mol Cancer Ther 7(9):2977-2987

83. Schoenlein PV et al (2009) Autophagy facilitates the progression of ERalpha-positive breast cancer cells to antiestrogen resistance. Autophagy 5(3):400-403

84. Duan L et al (2011) Prolylcarboxypeptidase regulates proliferation, autophagy, and resistance to 4-hydroxytamoxifen-induced cytotoxicity in estrogen receptor-positive breast cancer cells. J Biol Chem 286(4):2864-2876

85. Cook KL et al (2014) Hydroxychloroquine inhibits autophagy to potentiate antiestrogen responsiveness in ER + breast cancer. Clin Cancer Res 20(12):3222-3232
86. Hosokawa $\mathrm{N}$ et al (2009) Nutrient-dependent mTORC1 association with the ULK1-Atg13-FIP200 complex required for autophagy. Mol Biol Cell 20(7):1981-1991

87. Roop RP, Ma CX (2012) Endocrine resistance in breast cancer: molecular pathways and rational development of targeted therapies. Future Oncol 8(3):273-292

88. Paplomata E, O'Regan R (2014) The PI3K/AKT/mTOR pathway in breast cancer: targets, trials and biomarkers. Ther Adv Med Oncol 6(4):154-166

89. Lee JJ, Loh K, Yap YS (2015) PI3K/Akt/mTOR inhibitors in breast cancer. Cancer Biol Med 12(4):342-354

90. Polo ML et al (2015) Activation of PI3K/Akt/mTOR signaling in the tumor stroma drives endocrine therapy-dependent breast tumor regression. Oncotarget 6(26):22081-22097

91. Friedmann-Morvinski D, Verma IM (2014) Dedifferentiation and reprogramming: origins of cancer stem cells. EMBO Rep 15(3):244-253

92. Dean M (2005) Cancer stem cells: Implications for cancer causation and therapy resistance. Discov Med 5(27):278-282

93. Ben-Porath I et al (2008) An embryonic stem cell-like gene expression signature in poorly differentiated aggressive human tumors. Nat Genet 40(5):499-507

94. Yamanaka S, Blau HM (2010) Nuclear reprogramming to a pluripotent state by three approaches. Nature 465(7299):704-712

95. Corominas-Faja B et al (2013) Nuclear reprogramming of luminal-like breast cancer cells generates Sox2-overexpressing cancer stem-like cellular states harboring transcriptional activation of the mTOR pathway. Cell Cycle 12(18):3109-3124

96. Mou W et al (2015) Expression of Sox 2 in breast cancer cells promotes the recruitment of M2 macrophages to tumor microenvironment. Cancer Lett 358(2):115-123

97. Leung EY et al (2017) Endocrine therapy of estrogen receptorpositive breast cancer cells: early differential effects on stem cell markers. Front Oncol 7:184

98. Boyer LA et al (2005) Core transcriptional regulatory circuitry in human embryonic stem cells. Cell 122(6):947-956

99. Li X et al (2014) The transcriptional regulation of SOX2 on FOXA1 gene and its application in diagnosis of human breast and lung cancers. Clin Lab 60(6):909-918

100. Bora-Singhal N et al (2015) Gli1-mediated regulation of Sox2 facilitates self-renewal of stem-like cells and confers resistance to EGFR inhibitors in non-small cell lung cancer. Neoplasia 17(7):538-551

101. Flemban A, Qualtrough D (2015) The potential role of hedgehog signaling in the luminal/basal phenotype of breast epithelia and in breast cancer invasion and metastasis. Cancers (Basel) 7(3): 1863-1884

102. Shostak K, Chariot A (2011) NF-kappaB, stem cells and breast cancer: the links get stronger. Breast Cancer Res 13(4):214

103. Kotiyal S, Bhattacharya S (2014) Breast cancer stem cells, EMT and therapeutic targets. Biochem Biophys Res Commun 453(1):112-116 